\title{
Postablative thyroglobulin levels predict recurrence of thyroid cancer
}

Postablative thyroglobulin concentrations and localized tumor infiltration of adjoining tissues at the time of primary surgery are independent predictors of disease recurrence in patients with welldifferentiated thyroid cancer, report researchers from Finland.

In an observational, retrospective study, Pelttari et al. determined recurrence rates and cancer-specific deaths in 495 patients with stage I or II well-differentiated thyroid cancer, the majority of whom had been initially treated with both surgery and radioactive iodine remnant ablation, after a median follow-up of 16 years.

"Only a few studies have explored the possible role of postsurgery and/ or postabalative thyroglobulin levels in disease outcome," says lead author Hanna Pelttari (Helsinki University Central Hospital). Postoperative and postablative thyroglobulin levels were correlated with age, tumor size, local infiltration and nodal metastasis at the time of primary surgery.

A total of 51 patients (10.3\%)

experienced disease recurrence during follow-up; $84.3 \%$ of recurrences occurred within 10 years from diagnosis and initial treatment. Postablative thyroglobulin concentrations emerged as a strong predictor of recurrence, as did preoperative or microscopic evidence of local tissue infiltration by the tumor.

The researchers suggest that thyroglobulin may help to identify highrisk individuals among patients with well-differentiated thyroid cancer and thereby increase cost-effectiveness of the surveillance of this patient group.

Linda Koch

Original article Pelttari, H. et al. Post-ablative serum thyroglobulin is an independent predictor of recurrence in low-risk differentiated thyroid carcinoma-a 16-yr follow-up study. Eur. J. Endocrinol. 163,757-763 (2010) 